Original Research Paper

\title{
New Bivariate Exponentiated Modified Weibull Distribution
}

\author{
${ }^{1}$ Abdelfattah Mustafa and ${ }^{2}$ Mohamed A.W. Mahmoud \\ ${ }^{1}$ Department of Mathematics, Faculty of Science, Mansoura University, Mansoura 35516, Egypt \\ ${ }^{2}$ Department of Mathematics, Faculty of Science, Al-Azhar University, Nasr City 11884, Cairo, Egypt
}

Article history

Received: 05-08-2017

Revised: 19-09-2017

Accepted: 31-10-2017

Corresponding Author:

Abdelfattah Mustafa

Department of Mathematics,

Faculty of Science, Mansoura

University, Mansoura 35516,

Egypt

Email: abdelfatah_mustafa@yahoo.com

\begin{abstract}
The new modified Weibull distribution named exponentiated modified Weibull distribution introduced by many authors. In this study, we define and study a bivariate modified Weibull distribution. Some joint probability functions and conditional probability density function are obtained. Also, joint survival function is expressed in compact form. Several statistical properties of this distribution are discussed. In the area of stressstrength models, we estimate the reliability follow a bivariate exponentiated modified Weibull distribution with dependence between stress and strength variables. Parameters estimators using the maximum likelihood method are obtained. Moreover, a numerical illustration is used to obtain Maximum Likelihood Estimators (MLEs).
\end{abstract}

Keywords: Generalized Modified Weibull Distribution, Maximum Likelihood Estimators, Bivariate Distributions, Stress-Strength Models

\section{Introduction}

Sarhan and Zaindin (2009) introduced the new class from the Weibull distribution named modified Weibull distribution. The random variable $Y$ has univariate Modified Weibull (MW) distribution with parameters $a$, $b$ and $c$ if its Cumulative Distribution Function (CDF) has the following form:

$$
G_{M W}(y ; a, b, c)=1-\exp \left\{-\left(a y+b y^{c}\right)\right\}, y \geq 0
$$

where, $a, b, c>0$.

The Modified Weibull Distribution (MWD), generalizes several well- known distributions. Among of these distributions are the exponential distribution, the linear failure rate distribution, the Rayleigh distribution and the Weibull distribution.

The Exponentiated Modified Weibull Distribution (EMWD) introduced in Elbatal (2011). The random variable $Y$ has univariate EMWD with parameters $a, b, c$ and $v$ if its $\mathrm{CDF}$ is given as:

$G_{E M W}(y ; a, b, c, v)=\left(1-e^{-\left(a y+b y^{c}\right)}\right)^{v}, y \geq 0$

where, $v, a, b, c>0$.

This paper introduces a Bivariate Exponentiated Modified Weibull Distribution (BEMWD) by using the method of Marshall and Olkin (1986). Several bivariate distributions are derived by using this method, Marshall and Olkin (1986). Several bivariate exponential distribution introduced in Sarhan and Balakrishnan (2007). A new class of bivariate Gompertz distribution is introduced in Al-Khedhairi and El-Gohary (2011). The bivariate generalized exponential distribution proposed by Kundu and Gupta (2009), a new bivariate generalized Gompertz distribution presented in El-Sherpieny et al. (2013) and Marshall-Olkin bivariate Weibull distribution studied in Kundu and Gupta (2013); Mustafa (2016) introduced a new bivariate distribution with generalized quadratic hazard rate marginals.

The BMWD generalizes several distributions as following:

- The bivariate generalized exponential distribution, $\operatorname{BGED}(v, a)$, when $b \rightarrow 0$

- The bivariate exponentiated linear failure rate distribution, $\operatorname{BELFRD}(v, a, b)$, when $c=2$

- The bivariate exponentiated Rayleigh distribution, $\operatorname{BERD}(v, b)$, when $a \rightarrow 0, c=2$

- The bivariate exponentiated Weibull distribution, $\operatorname{BEWD}(v, a, b)$, when $a \rightarrow 0$

The rest of the paper is organized as follows. Some properties of the BGQHRD are presented in Section 2. Section 3, presents the reliability analysis. Section 4 , gives the stress-strength model. The parameter estimations using maximum likelihood is given in Section 5. We use a set of 
real data in Section 6 as an application. Conclusions for the article is introduced in Section 7.

\section{A New Bivariate Exponentiated Modified Weibull Distribution}

In this section, we discuss the new Bivariate Exponentiated Modified Weibull Distribution (BEMWD). We start with the joint cumulative distribution function of the distribution.

Suppose $V_{1}, V_{2}$ and $V_{3}$ are three independent random variables such that $V_{i} \sim \operatorname{EMW}\left(v_{i}, a, b, c\right)$ for $i=1,2,3$.

Define:

$$
Y_{1}=\max \left(V_{1}, V_{3}\right), Y_{2}=\max \left(V_{2}, V_{3}\right)
$$

\section{Theorem 1}

The joint cumulative distribution function of $Y_{1}, Y_{2}$ is:

$G_{Y_{1}, Y_{2}}\left(y_{1}, y_{2}\right)=P\left[Y_{1} \leq y_{1}, Y_{2} \leq y_{2}\right]$

$=\prod_{i=1}^{3} G_{E M W}\left(y_{i} ; v_{i}, a, b, c\right)$

where, $y_{3}=\min \left(y_{1}, y_{2}\right)$

Proof

The joint cumulative distribution function is defined as:

$$
\begin{aligned}
& G\left(y_{1}, y_{2}\right)=P\left[Y_{1} \leq y_{1}, Y_{2} \leq y_{2}\right] \\
& =P\left[\max \left(V_{1}, V_{3}\right) \leq y_{1}, \max \left(V_{2}, V_{3}\right) \leq y_{2}\right] \\
& =P\left[V_{1} \leq y_{1}, V_{2} \leq y_{2}, V_{3} \leq \min \left(y_{1}, y_{2}\right)\right]
\end{aligned}
$$

But the random variables $V_{1}, V_{2}$ and $V_{3}$ are mutually independent, then:

$$
\begin{gathered}
G\left(y_{1}, y_{2}\right)=P\left[V_{1} \leq y_{1}\right] P\left[V_{2} \leq y_{2}\right] P\left[V_{3} \leq \min \left(y_{1}, y_{2}\right)\right] \\
=\prod_{i=1}^{3} G_{E M W}\left(y_{i} ; v_{i}, a, b, c\right)
\end{gathered}
$$

This completes the proof.

The joint PDF of the BEMW can be obtained as the following theorem.

\section{Theorem 2}

If $\left(Y_{1}, Y_{2}\right) \sim B E M W\left(v_{1}, v_{2}, v_{3}, a, b, c\right)$, then the joint $\operatorname{PDF}$ of $\left(Y_{1}, Y_{2}\right)$ is give by:

$$
g_{Y_{1}, Y_{2}}\left(y_{1}, y_{2}\right)=\left\{\begin{array}{ccc}
g_{1}\left(y_{1}, y_{2}\right) & \text { if } & y_{1}<y_{2} \\
g_{2}\left(y_{1}, y_{2}\right) & \text { if } & y_{2}<y_{1} \\
g_{3}(y, y) & \text { if } & y_{1}=y_{2}=y
\end{array}\right.
$$

Where:

$$
\begin{aligned}
& g_{1}\left(y_{1}, y_{2}\right)=g_{E M W}\left(y_{2} ; v_{2}, a, b, c\right) g_{E M W}\left(y_{1} ; v_{1}+v_{3}, a, b, c\right) \\
& g_{2}\left(y_{1}, y_{2}\right)=g_{E M W}\left(y_{1} ; v_{1}, a, b, c\right) g_{E M W}\left(y_{2} ; v_{2}+v_{3}, a, b, c\right) \\
& g_{3}(y, y)=\left(\frac{v_{3}}{v_{1}+v_{2}+v_{3}}\right) g_{E M W}\left(y ; v_{1}+v_{2}+v_{3}, a, b, c\right)
\end{aligned}
$$

\section{Proof}

We can derive the expression for $g_{1}\left(y_{1}, y_{2}\right)$ and $g_{2}\left(y_{1}, y_{2}\right)$ by differentiating the joint CDF given in Equation (3) with respect to $y_{1}$ and $y_{2}$. But can be derived by using the following identity:

$$
\begin{aligned}
& \int_{0}^{\infty} \int_{0}^{y_{2}} g_{1}\left(y_{1}, y_{2}\right) d y_{1} d y_{2}+\int_{0}^{\infty} \int_{0}^{y_{1}} g_{2}\left(y_{1}, y_{2}\right) d y_{2} d y_{1} \\
& \quad+\int_{0}^{\infty} g_{3}(y, y) d y=1
\end{aligned}
$$

Then, we can find mathematically that:

$$
\begin{aligned}
& I_{1}=\int_{0}^{\infty} \int_{0}^{y_{2}} g_{1}\left(y_{1}, y_{2}\right) d y_{1} d y_{2} \\
& =\int_{0}^{\infty} v_{2}\left(a+b c y_{2}^{c-1}\right) e^{-\left(a y_{2}+b y_{2}^{c}\right)}\left[1-e^{-\left(a y_{2}+b y_{2}^{c}\right)}\right]^{v_{1}+v_{2}+v_{3}-1} d y_{2} .
\end{aligned}
$$

Similarly:

$$
\begin{aligned}
& I_{2}=\int_{0}^{\infty} \int_{0}^{y_{1}} g_{2}\left(y_{1}, y_{2}\right) d y_{2} d y_{1} \\
& =\int_{0}^{\infty} v_{1}\left(a+b c y_{1}^{c-1}\right) e^{-\left(a y_{1}+b y_{1}^{c}\right)}\left[1-e^{-\left(a y_{1}+b y_{1}^{c}\right)}\right]^{v_{1}+v_{2}+v_{3}-1} d y_{1} .
\end{aligned}
$$

From Equations (9) and (10), we then get:

$$
\begin{aligned}
& \int_{0}^{\infty} g_{3}(y, y) d y=1-I_{1}-I_{2} \\
& =\int_{0}^{\infty} v \cdot\left(a+b c y^{c-1}\right) e^{-\left(a y+b y^{c}\right)}\left[1-e^{-\left(a y+b y^{c}\right)}\right]^{\nu,-1} d x \\
& -\int_{0}^{\infty} v_{2}\left(a+b c y^{c-1}\right) e^{-\left(a y+b y^{c}\right)}\left[1-e^{-\left(a y+b y^{c}\right)}\right]^{\nu-1} d y \\
& -\int_{0}^{\infty} v_{1}\left(a+b c y^{c-1}\right) e^{-\left(a y+b y^{c}\right)}\left[1-e^{-\left(a y+b y^{c}\right)}\right]^{\nu-1} d y \\
& =\int_{0}^{\infty} v_{3}\left(a+b c y^{c-1}\right) e^{-\left(a y+b y^{c}\right)}\left[1-e^{-\left(a y+b y^{c}\right)}\right]^{\nu-1} d y .
\end{aligned}
$$

where, $v_{.}=v_{1}+v_{2}+v_{3}$.

That is:

$g_{3}(y, y)=v_{3}\left(a+b c x^{c-1}\right) e^{-\left(a y+b y^{c}\right)}\left[1-e^{-\left(a y+b y^{c}\right)}\right]^{\nu_{1}+v_{2}+v_{3}-1}$

The proof is completed.

The marginal density function of $Y_{i}, i=1,2$ can be obtained as follows. 


\section{Theorem 3}

The marginal PDF of $Y_{i} i=1,2$ is given by:

$g_{Y i}\left(y_{i}\right)=g_{E M W}\left(y_{i} ; v_{i}+v_{3}, a, b, c\right)$

where, $v_{i}, a, b, c>0, i=1,2$.

\section{Proof}

The marginal CDF of $Y_{i} i=1,2$, say $F_{Y_{i}}\left(y_{i}\right)$, is:

$$
\begin{aligned}
& G_{Y_{i}}\left(y_{i}\right)=P\left[Y_{i} \leq y_{i}\right]=P\left[\max \left(V_{i}, V_{3}\right) \leq y_{i}\right] \\
& =P\left[V_{i} \leq y_{i}, V_{3} \leq y_{i}\right]
\end{aligned}
$$

Since $V_{i}$ and $V_{3}$ are independent, we get:

$G_{Y_{i}}\left(y_{i}\right)=P\left[Y_{i} \leq y_{i}\right] P\left[V_{3} \leq y_{i}\right]=\left[1-e^{-\left(a y_{i}+b y_{i}^{c}\right)}\right]^{v_{i}+v_{3}}$

$=G_{E M W}\left(y_{i} ; v_{i}+v_{3}, a, b, c\right)$

The formula given in Equation (12) can be obtained by differentiating Equation (13) with respect to $y_{i}$.

That is, $Y_{i} \sim \operatorname{EMWD}\left(v_{i}+v_{3}, a, b, c\right)$.

The following theorem gives the rth moments of $Y_{i}, i$ $=1,2$.

\section{Theorem 4}

The rth moment of $Y_{i}(i=1,2)$ derived by:

$$
\begin{aligned}
& \mu_{r}^{\prime}=E\left[Y_{i}^{r}\right]=\left(v_{i}+v_{3}\right) \sum_{j=0}^{v_{i}+v_{3}-1} \sum_{k=0}^{\infty}\left(\begin{array}{c}
v_{i}+v_{3}-1 \\
j
\end{array}\right) \frac{(-1)^{j+k} \beta^{k}}{k ! a^{k c+r+1}} \\
& (j+1)^{(1-c) k-r-1}\left[a \Gamma(k c+r+1)+\frac{b c \Gamma((k+1) \gamma+r)}{[(j+1) a]^{c-1}}\right] .
\end{aligned}
$$

\section{Proof}

Starting with:

$$
\mu_{r}^{\prime}=E\left[Y_{i}^{r}\right]=\int_{0}^{\infty} y_{i}^{r} g_{Y_{i}}\left(y_{i}\right) d y_{i}
$$

and from Equation (12), we get:

$$
\begin{aligned}
& E\left[Y_{i}^{r}\right]=\left(v_{i}+v_{3}\right) \int_{0}^{\infty} y_{i}^{r}\left(a+b c y_{i}^{c-1}\right) e^{-\left(a y_{i}+b y_{i}^{c}\right)} \\
& {\left[1-e^{-\left(a y_{i}+b y_{i}^{c}\right)}\right]^{v_{i}+v_{3}-1} d y_{i} .}
\end{aligned}
$$

Since $0<e^{-\left(a y_{i}+b y_{i}^{c}\right)}<1$, for $y>0$ then using the binomial expansion of $\left[1-e^{-\left(a y_{i}+b y_{i}^{c}\right)}\right]^{\nu_{i}+v_{3}-1}$ given by:

$$
\left[1-e^{-\left(a y_{i}+b y_{i}^{c}\right)}\right]^{v_{i}+v_{3}-1}=\sum_{j=0}^{v_{i}+v_{3}-1}\left(\begin{array}{c}
v_{i}+v_{3}-1 \\
j
\end{array}\right)(-1)^{j} e^{-j\left(a y_{i}+b y_{i}^{c}\right)} .
$$

Substituting from Equation (16) into (15), we get:

$$
\begin{aligned}
E\left[Y_{i}^{r}\right]= & \left(v_{i}+v_{3}\right) \sum_{j=0}^{v_{i}+v_{3}-1}\left(\begin{array}{c}
v_{i}+v_{3}-1 \\
j
\end{array}\right)(-1)^{j} \times \\
& \int_{0}^{\infty} y_{i}^{r}\left(a+b c y_{i}^{c-1}\right) e^{-(j+1)\left(a y_{i}+b y_{i}^{c}\right)} d y_{i} .
\end{aligned}
$$

Using the series expansion of $e^{-(j+1) b y_{i}^{c}}$, one gets:

$$
\begin{aligned}
E\left[Y_{i}{ }^{r}\right]= & \left(v_{i}+v_{3}\right) \sum_{j=0}^{v_{i}+v_{3}-1} \sum_{k=0}^{\infty}\left(\begin{array}{c}
v_{i}+v_{3}-1 \\
j
\end{array}\right) \frac{(-1)^{j+k}[(j+1) b]^{k}}{k !} \\
& \int_{0}^{\infty}\left(a+b c y_{i}^{c-1}\right) y_{i}^{k c+r} e^{-(j+1) a y_{i}} d y_{i} \\
= & \left(v_{i}+v_{3}\right) \sum_{j=0}^{v_{i}+v_{3}-1} \sum_{k=0}^{\infty}\left(\begin{array}{c}
v_{i}+v_{3}-1 \\
j
\end{array}\right) \frac{(-1)^{j+k}[(j+1) b]^{k}}{k !} \\
\int_{0}^{\infty}\left[a y_{i}^{k c+r}+b c y_{i}{ }^{(k+1) c+r-1}\right] & e^{-(j+1) a y_{i}} d y_{i}
\end{aligned}
$$

Let $v=(j+1) \alpha y_{i}$, in the above integral, then we can get:

$$
\begin{aligned}
& E\left[Y_{i}{ }^{r}\right]=\left(v_{i}+v_{3}\right) \sum_{j=0}^{v_{i}+v_{3}-1} \sum_{k=0}^{\infty}\left(\begin{array}{c}
v_{i}+v_{3}-1 \\
j
\end{array}\right) \frac{(-1)^{j+k}[(j+1) b]^{k}}{k ![(j+1) a]^{k c+r+1}} \\
& {\left[a \Gamma(k c+r+1)+\frac{b c \Gamma((k+1) c+r)}{[(j+1) a]^{c-1}}\right]}
\end{aligned}
$$

Thus (14) is obtained.

The following theorem gives the conditional PDF.

\section{Theorem 5}

The conditional probability density function of $Y_{i}$, give $Y_{j}=y_{j}$ is given by:

$g_{Y_{i} \mid Y_{j}}\left(y_{i} \mid y_{j}\right)= \begin{cases}g_{Y_{i} \mid Y_{j}}^{(1)}\left(y_{i} \mid y_{j}\right) & \text { if } y_{i}<y_{j} \\ g_{Y_{i} \mid Y_{j}}^{(2)}\left(y_{i} \mid y_{j}\right) & \text { if } y_{j}<y_{i} \\ g_{Y_{i} \mid Y_{j}}^{(3)}\left(y_{i} \mid y_{j}\right) & \text { if } y_{i}<y_{j}\end{cases}$

where:

$$
\begin{aligned}
& g_{Y_{i} Y_{j}}^{(1)}\left(y_{i} \mid y_{j}\right)=\left(\frac{v_{j}}{v_{j}+v_{3}}\right) \frac{g_{Y_{i}}\left(y_{i}, v_{i}+v_{3}, a, b, c\right)}{G_{Y_{j}}\left(y_{j} ; v_{3}, a, b, c\right)} \\
& =\frac{1}{\left(v_{j}+v_{3}\right)\left[1-e^{-\left(a y_{j}+b y_{j}^{c}\right)}\right]^{v_{3}}}\left[v_{j}\left(v_{i}+v_{3}\right)\right.
\end{aligned}
$$

$\left.\left(a+b c y_{i}^{c-1}\right) e^{-\left(a y_{i}+b y_{i}^{c}\right)}\left[1-e^{-\left(a y_{i}+b y_{i}^{c}\right)}\right] v_{i}^{v_{i}+v_{3}-1}\right]$

$$
\begin{aligned}
& g_{Y_{i} Y_{j}}^{(2)}\left(y_{i} \mid y_{j}\right)=g_{Y_{i}}\left(y_{i} ; v_{i}, a, b, c\right) \\
& =v_{i}\left(a+b c y_{i}^{c-1}\right)-e^{-\left(a y_{i}+b y_{i}^{c}\right)}\left[-e^{-\left(a y_{i}+b y_{i}^{c}\right)}\right]^{v_{i}-1}
\end{aligned}
$$




$$
\begin{aligned}
g_{Y_{i} Y_{j}}^{(3)}\left(y_{i} \mid y_{j}\right) & =\left(\frac{v_{3}}{v_{j}+v_{3}}\right) G_{Y_{i}}\left(y_{i} ; v_{i}, a, b, c\right) \\
& =\left(\frac{v_{3}}{v_{j}+v_{3}}\right)\left[1-e^{-\left(a y_{i}+b y_{i}^{c}\right)}\right]^{v_{i}} .
\end{aligned}
$$

\section{Proof}

Since the conditional PDF is given by:

$$
g_{Y_{i} \mid Y_{j}}\left(y_{i} \mid y_{j}\right)=\frac{g_{Y_{i} Y_{j}}\left(y_{i} y_{j}\right)}{g_{Y_{j}}\left(y_{j}\right)}
$$

The theorem can be proved by substituting form Equations (5) and (12), in the above relation.

\section{Reliability Analysis}

The joint survival function of $\left(Y_{1}, Y_{2}\right), \mathrm{CDF}$ of the random variables $Z=\max \left\{Y_{1}, Y_{2}\right\}$ and $W=\min \left\{Y_{1}, Y_{2}\right\}$ can be derived in this section. The random variable $Z=$ $\max \left\{Y_{1}, Y_{2}\right\}$ represents the lifetime for a parallel system with two components and $W=\min \left\{Y_{1}, Y_{2}\right\}$ is the lifetime of a series system with two components.

\section{Theorem 6}

The joint survival function of $\left(Y_{1}, Y_{2}\right)$ is obtained as:

$$
S_{Y_{1}, Y_{2}}\left(y_{1}, y_{2}\right)= \begin{cases}S_{1}\left(y_{1}, y_{2}\right) & \text { if } y_{1}<y_{2} \\ S_{2}\left(y_{1}, y_{2}\right) & \text { if } y_{2}<y_{1} \\ S_{0}\left(y_{1}, y_{2}\right) & \text { if } y_{1}=y_{2}=y\end{cases}
$$

where:

$$
\begin{aligned}
& S_{1}\left(y_{1}, y_{2}\right)=1-\left[1-e^{-\left(a y_{2}+b y_{2}^{c}\right)}\right]^{\nu_{2}+v_{3}} \\
& -\left[1-e^{-\left(a y_{1}+b y_{1}^{c}\right)}\right]^{\nu_{1}+v_{3}} \times\left(1-\left[1-e^{-\left(a y_{2}+b x_{2}^{c}\right)}\right]^{\nu_{2}}\right), \\
& S_{2}\left(y_{1}, y_{2}\right)=1-\left[1-e^{-\left(a y_{1}+b y y_{1}^{c}\right)}\right]^{\nu_{1}+\nu_{2}} \\
& -\left[1-e^{-\left(a y_{2}+b y_{2}^{c}\right)}\right]^{\nu_{2}+v_{3}} \times\left(1-\left[1-e^{-\left(a y_{1}+b x_{1}^{c}\right)}\right]^{\nu_{1}}\right), \\
& S_{0}(y, y)=1-\left[1-e^{-\left(a y+b y^{c}\right)}\right]^{\nu_{3}}\left(1-\left[1-e^{-\left(a y+b y^{c}\right)}\right]^{\nu_{1}}+\right. \\
& \left.\left[1-e^{-\left(a y+b y^{c}\right)}\right]^{\nu_{2}}\left[1-e^{-\left(a y+b y^{c}\right)}\right]^{\nu_{1}+v_{2}}\right) .
\end{aligned}
$$

\section{Proof}

One can obtain Equation (21) by using the relation:

$$
S_{Y_{1} Y_{2}}\left(y_{1}, y_{2}\right)=1-G_{Y_{1}}\left(y_{1}\right)-G_{Y_{2}}\left(y_{2}\right)+G_{Y_{1} Y_{2}}\left(y_{1}, y_{2}\right)
$$

Substituting from Equations (3) and (13) into Equation (22), we can obtain Equation (21). This completes the proof.

Also, the bivariate failure rate function can be obtained by substituting from Equations (5) and (21) in the following relation, Basu (1971); Johnson and Kotz (1975):

$h_{Y_{1}, Y_{2}}\left(y_{1}, y_{2}\right)=\frac{g_{Y_{1}, Y_{2}}\left(y_{1}, y_{2}\right)}{S_{Y_{1}, Y_{2}}\left(y_{1}, y_{2}\right)}$.

\section{Lemma 1.}

The random variable $Z=\max \left\{Y_{1}, Y_{2}\right\}$ following CDF:

$$
G_{Z}(z)=\left[1-e^{-\left(a z+b z^{c}\right)}\right]^{v_{1}+v_{2}+v_{3}}
$$

Proof

Since:

$$
\begin{aligned}
& G_{Z}(z)=P[Z \leq z]=P\left[\max \left\{Y_{1}, Y_{2}\right\} \leq z\right] \\
& =P\left[Y_{1} \leq z, Y_{2} \leq z\right] \\
& =P\left[\max \left\{V_{1}, V_{3}\right\} \leq z, \max \left\{V_{2}, V_{3}\right\} \leq z\right] \\
& =P\left[V_{1}, \leq z, V_{2} \leq z, V_{3} \leq z\right]
\end{aligned}
$$

But $V_{1}, V_{2}$ and $V_{3}$ are independent random variables, so:

$$
\begin{aligned}
& G_{Z}(z)=P\left[V_{1} \leq z\right] P\left[V_{2} \leq z\right] P\left[V_{3} \leq z\right] \\
& =\prod_{i=1}^{3} G_{E M W}\left(z ; v_{i}, a, b, c\right)
\end{aligned}
$$

From Equation (2) into (25), we get (24).

We note that, $Z \sim E M W D\left(v_{1}+v_{2}+v_{3}, a, b, \mathrm{c}\right)$.

\section{Lemma 2}

Let $W=\min \left\{Y_{1}, Y_{2}\right\}$ be the random variable, the $G_{W}(w)$ can be derived as:

$$
\begin{aligned}
& G_{W}(w)=\left[1-e^{-\left(a w+b w^{c}\right)}\right]^{\nu_{1}}+\left[1-e^{-\left(a w+b w^{c}\right)}\right]^{\nu_{2}} \\
& -\left[1-e^{-\left(a w+b w^{c}\right)}\right]^{\nu_{1}+v_{2}+\nu_{3}}
\end{aligned}
$$

\section{Proof}

Since:

$$
\begin{aligned}
& G_{W}(w)=P[W \leq w]=P\left[\min \left\{Y_{1}, Y_{2}\right\} \leq w\right] \\
& =1-P\left[\min \left\{Y_{1}, Y_{2}\right\}>w\right] \\
& =1-P\left[Y_{1}>w, Y_{2}>w\right]=1-S(w, w] .
\end{aligned}
$$

Substituting from Equation (22) into Equation (27), we get: 


$$
G_{w}(w)=G_{Y_{1}}(w)+G_{Y_{2}}(w)-G_{Y_{1}, Y_{2}}(w, w)
$$

We can obtain Equation (26) by substituting from Equations (3) and (13) into Equation (28).

\section{Stress-Strength Model}

In this section, we consider the problem of the stressstrength model. The reliability $R=P\left[Y_{1}<Y_{2}\right]$ which defined as a life of a component which has a random variable strength $Y_{2}$ and is subjected to random stress $Y_{1}$. We derive the form of $R$ when $\left(Y_{1}, Y_{2}\right)$ follow a $\operatorname{BEM}\left(v_{1}\right.$, $\left.v_{2}, v_{3}, a, b, c\right)$ with dependence between $Y_{1}$ and $Y_{2}$.

\section{Theorem 7.}

For the random variables $\left(Y_{1}, Y_{2}\right)$ with BEMW, then $R=P\left(Y_{1}<Y_{2}\right)$ is:

$$
\begin{aligned}
& R=v_{2}\left(v_{1}+v_{3}\right) \sum_{i=0}^{v_{2}-1} \sum_{j=0}^{\infty} \sum_{k=0}^{v_{1}+v_{2}-1} \sum_{\ell=0}^{\infty}\left(\begin{array}{c}
v_{2}-1 \\
i
\end{array}\right) \times \\
& \left(\begin{array}{c}
v_{1}+v_{3}-1 \\
k
\end{array}\right) \frac{(-1)^{i+j+k+\ell}(k+1)^{\ell} b^{j+\ell}}{j ! \ell ! a^{j c+1}(i+1)^{j(c-1)+1}} \times \\
& {\left[a(j c) ! \sum_{m=0}^{j c} \psi_{m}+\frac{b c[(j+1) c-1] !^{(j+1) c-1}}{[(i+1) a]^{c-1}} \sum_{m=0} \psi_{m}\right],}
\end{aligned}
$$

where:

$$
\begin{aligned}
& \psi_{m}=\frac{[(i+1) a]^{m}}{m ![(k+i+2) a]^{m+\ell c+1}} \times \\
& {\left[a \Gamma(m+\ell c+1)+\frac{b c \Gamma(m+(\ell+1) c)}{[(k+i+2) a]^{c-1}}\right] .}
\end{aligned}
$$

\section{Proof}

Since:

$$
R=P\left(Y_{1}<Y_{2}\right)=\int_{0}^{\infty} \int_{y_{1}}^{\infty} g\left(y_{1}, y_{2}\right) d y_{2} d y_{1}
$$

Substituting from Equation (6) into Equation (30), we get:

$$
\begin{aligned}
& R=\int_{0}^{\infty} \int_{y_{1}}^{\infty} v_{2}\left(a+b c y_{2}^{c-1}\right) e^{-\left(a y_{2}+b y_{1}^{c}\right)}\left[1-e^{-\left(a y_{2}+b y_{2}^{c}\right)}\right]^{v_{2}-1} \times \\
& \left(v_{1}+v_{3}\right)\left(a+b c y_{2}^{c-1}\right) e^{-\left(a y_{1}+b y_{1}^{c}\right)}\left[1-e^{-\left(a y_{1}+b y_{1}^{c}\right)}\right]^{v_{1}+v_{3}-1} d y_{2} d y_{1} . \\
& \text { By using the binomial expansion for } \\
& {\left[1-e^{-\left(a y_{2}+b y y_{2}^{c}\right)}\right]^{v_{2}-1} \text {, we get: }} \\
& R=\sum_{i=0}^{v_{2}-1}\left(\begin{array}{c}
v_{2}-1 \\
i
\end{array}\right)(-1)^{i} v_{2}\left(v_{1}+v_{3}\right) \int_{0}^{\infty} \int_{y_{1}}^{\infty}\left(a+b c y_{2}^{c-1}\right) e^{-(i+1)\left(a y_{2}+b y_{2}^{c}\right)} \\
& \left(a+b c y_{1}^{c-1}\right) e^{-\left(a y_{1}+b y_{1}^{c}\right)}\left[1-e^{-\left(a y_{1}+b y_{1}^{c}\right)}\right]^{v_{1}+v_{3}-1} d y_{2} d y_{1}
\end{aligned}
$$

Using the series expansion for $e^{-(i+1) b y_{2}^{c}}$, we have:

$$
\begin{aligned}
& R=v_{2}\left(v_{1}+v_{3}\right) \sum_{i=0}^{v_{2}-1} \sum_{j=0}^{\infty}\left(\begin{array}{c}
v_{2}-1 \\
i
\end{array}\right)(-1)^{i+j} \frac{[(i+1) b]^{j}}{j !} \times \\
& \int_{0}^{\infty}\left(a+b c y_{2}^{c-1}\right) e^{-\left(a y_{1}+b y_{1}^{c}\right)}\left[1-e^{-\left(a y_{1}+b y_{1}^{c}\right)}\right]^{v_{1}+v_{3}-1} \times \\
& \int_{y_{1}}^{\infty}\left(a y_{2}^{c j}+b c y_{2}^{c(j+1)-1}\right) e^{-(i+1) a y_{2}} d y_{2} d y_{1}
\end{aligned}
$$

Let $u=(i+1) a y_{2}$, then from Equation (31), we get:

$$
\begin{aligned}
& R=v_{2}\left(v_{1}+v_{3}\right) \sum_{i=0}^{v_{2}-1} \sum_{j=0}^{\infty}\left(\begin{array}{c}
v_{2}-1 \\
i
\end{array}\right) \frac{(-1)^{i+j}}{j !}[(i+1) b]^{j} \\
& \int_{0}^{\infty}\left(a+b c y_{1}^{c-1}\right) e^{-\left(a y_{1}+b y_{1}^{c}\right)}\left[1-e^{-\left(a y_{1}+b y_{1}^{c}\right)}\right]^{v_{1}+v_{3}-1} \\
& {\left[\frac{a \Gamma\left(c j+1,(i+1) a y_{1}\right)}{[(i+1) a]^{j c+1}}+\frac{b c \Gamma\left(c(j+1),(i+1) a y_{1}\right)}{[(i+1) a]^{(j+1) c}}\right] d y_{1}}
\end{aligned}
$$

where, $\Gamma(s, x)$ is the incomplete Gamma function:

$$
\Gamma(s, x)=\int_{x}^{\infty} t^{s-1} e^{-t} d t
$$

Since $0<e^{-\left(a y_{1}+b y_{1}^{c}\right)}<1$, by using binomial expansion:

$$
\left[1-e^{-\left(a y_{1}+b y y_{1}^{c}\right)}\right]^{v_{1}+v_{3}-1}=\sum_{k=0}^{v_{1}+v_{3}-1}\left(\begin{array}{c}
v_{1}+v_{3}-1 \\
k
\end{array}\right)(-1)^{k} e^{-k\left(a y_{1}+b y y_{1}^{c}\right)} .
$$

Substituting from Equation (34) into Equation (32), we get:

$$
\begin{aligned}
& R=v_{2}\left(v_{1}+v_{3}\right) \sum_{i=0}^{v_{2}-1} \sum_{j=0}^{\infty} \sum_{k=0}^{v_{1}+v_{3}-1}\left(\begin{array}{c}
v_{2}-1 \\
i
\end{array}\right)\left(\begin{array}{c}
v_{1}+v_{3}-1 \\
k
\end{array}\right)(-1)^{i+j+k} \times \\
& \frac{[(i+1) b]^{j}}{j !(i+1) a]^{j c+1}} \int_{0}^{\infty}\left(a+b c y_{1}^{c-1}\right) e^{-(k+1)\left(a y_{1}+b y_{1}^{c}\right)} \times \\
& {\left[a \Gamma\left(c j+1,(i+1) a y_{1}\right)+\frac{b c \Gamma\left(c(j+1),(i+1) a y_{1}\right)}{[(i+1) a]^{c-1}}\right] d y_{1} .}
\end{aligned}
$$

Since $0<e^{-(k+1) b y_{1}^{c}}<1$, by using the series expansion, then:

$$
\begin{aligned}
& R=v_{2}\left(v_{1}+v_{3}\right) \times \sum_{i=0}^{v_{2}-1} \sum_{j=0}^{\infty} \sum_{k=0}^{v_{1}+v_{3}-1} \sum_{\ell=0}^{\infty}\left(\begin{array}{c}
v_{2}-1 \\
i
\end{array}\right)\left(\begin{array}{c}
v_{1}+v_{3}-1 \\
k
\end{array}\right) \times \\
& \frac{(-1)^{i+j+k+\ell}(k+1)^{\ell} b^{j+\ell}}{j ! \ell ! a^{j c+1}(i+1)^{j(c-1)+1}}\left[a I_{1}+\frac{b c}{[(i+1) a]^{c-1}} I_{2}\right]
\end{aligned}
$$

where:

$$
I_{1}=\int_{0}^{\infty}\left(a+b c y_{1}^{c-1}\right) y_{1}^{\ell c} e^{-(k+1) a y_{1}} \Gamma\left(j c+1,(i+1) a y_{1}\right) d y_{1},
$$




$$
I_{2}=\int_{0}^{\infty}\left(a+b c y_{1}^{c-1}\right) y_{1}^{\ell c} e^{-(k+1) a y_{1}} \Gamma\left((j+1) c,(i+1) a y_{1}\right) d y_{1} .
$$

If $c$ is an integer then by using the following identity:

$$
\Gamma\left((n, z)=(n-1) ! e^{-z} \sum_{m=0}^{n-1} \frac{z^{m}}{m !}\right.
$$

One can evaluate $I_{1}$ and $I_{2}$ as follows:

$$
\begin{aligned}
& I_{1}=(j c) ! \sum_{m=0}^{j c} \frac{[(i+1) a]^{m}}{m ![(k+i+2) a]^{m+\ell c+1}} \times \\
& {\left[a \Gamma(m+\ell c+1)+\frac{b c \Gamma(m+(\ell+1) c)}{[(k+i+2) a]^{c-1}}\right]=(j c) ! \sum_{m=0}^{j c} \psi_{m},}
\end{aligned}
$$

where:

$$
\begin{aligned}
\psi_{m}= & \frac{[(i+1) a]^{m}}{m ![(k+i+2) a]^{m+\ell c+1}} \times \\
& {\left[a \Gamma(m+\ell c+1)+\frac{b c \Gamma(m+(\ell+1) c)}{[(k+i+2) a]^{c-1}}\right] }
\end{aligned}
$$

and

$$
I_{2}=((j+1) c-1) ! \sum_{m=0}^{(j+1) c-1} \psi_{m}
$$

Substituting from Equations (39) and (40) into Equation (35), we get:

$$
\begin{aligned}
& R=v_{2}\left(v_{1}+v_{3}\right) \sum_{i=0}^{v_{2}-1} \sum_{j=0}^{\infty} \sum_{k=0}^{v_{1}+v_{3}-1} \sum_{\ell=0}^{\infty}\left(\begin{array}{c}
v_{2}-1 \\
i
\end{array}\right)\left(\begin{array}{c}
v_{1}+v_{3}-1 \\
k
\end{array}\right) \times \\
& \frac{(-1)^{i+j+k+\ell}(k+1)^{\ell} b^{j+\ell}}{j ! \ell ! a^{j c+1}(i+1)^{j(c-1)+1}} \times \\
& {\left[a(j c) ! \sum_{m=0}^{j c} \psi_{m}+\frac{b c[(j+1) c-1]}{\left[(i+1)_{a}\right]^{c-1}} \sum_{m=0}^{(j+1) c-1} \psi_{m}\right]}
\end{aligned}
$$

This completes the proof.

\section{Maximum Likelihood Estimators}

We consider constant values to the parameters $a=$ 0.012 and $b=2.159 \times 10^{-8}$, (Sarhan and Zaindin, 2009). We want to estimate the unknown parameters $v_{1}, v_{2}, v_{3}$ and $c$ of the BEMWD. The maximum likelihood method can be used to estimate these unknown parameters.

Let $\left.\left(\left(y_{11}, y_{21}\right)\right),\left(y_{12}, y_{22}\right), \ldots,\left(y_{1 m}, y_{2 m}\right)\right)$ be a random sample from $\operatorname{BEMW}\left(v_{1}, v_{2}, v_{3}, a, b, c\right)$, suppose the following notation:

$$
\begin{array}{cc}
m_{1}=\left(i ; y_{1 i}<y_{2 i}\right), & m_{2}=\left(i ; y_{1 i}>y_{2 i}\right) \\
m_{3}=\left(i ; y_{1 i}=y_{2 i}=y_{i}\right), & m=m_{1}+m_{2}+m_{3} .
\end{array}
$$

For the sample of size $m$, the likelihood function is given by:

$$
L(\Phi)=\prod_{i=1}^{m_{1}} g_{1}\left(y_{1 i}, y_{2 i}\right) \prod_{i=1}^{m_{2}} g_{2}\left(y_{1 i}, y_{2 i}\right) \prod_{i=1}^{m_{3}} g_{3}\left(y_{i}, y_{i}\right)
$$

where, $\Phi=\left(v_{1}, v_{2}, v_{3}, a, b, c\right)$.

Substituting from Equations $(6,7)$ and (8) into Equation (42), we get:

$$
\begin{aligned}
& L(\Phi)=\prod_{i=1}^{m_{1}}\left\{v_{2}\left(a+b c y_{2}^{c-1}\right) e^{-\left(a y_{2 i}+b y y_{2 i}^{c}\right)}\left[1-e^{-\left(a y_{2 i}+b y y_{2 i}\right)}\right]^{v_{2}-1}\right. \\
& \left.\left(v_{1}+v_{3}\right)\left(a+b c y_{1 i}^{c-1}\right) e^{-\left(a y_{1 i}+b y_{1 i}^{c}\right)}\left[1-e^{-\left(a y_{1 i}+b y_{1 i}^{c}\right)}\right]^{v_{1}+v_{3}-1}\right\} \\
& \prod_{i=1}^{m_{2}}\left\{v_{1}\left(a+b c y_{1 i}^{c-1}\right) e^{-\left(a y_{1 i}+b y y_{1 i}^{c}\right)}\left[1-e^{-\left(a y_{1 i}+b y_{i i}^{c}\right)}\right]^{v_{1}-1}\left(v_{2}+v_{3}\right)\right. \\
& \left.\left(a+b c y_{2 i}^{c-1}\right) e^{-\left(a y_{2 i}+b y_{2 i}\right)}\left[1-e^{-\left(a y_{2 i}+b y_{2 i}^{c}\right)}\right]^{\nu_{2}+\nu_{3}-1}\right\} \\
& \prod_{i=1}^{m_{3}}\left\{v_{3}\left(a+b c y_{i}^{c-1}\right) e^{-\left(a y_{i}+b y_{i}^{c}\right)}\left[1-e^{-\left(a y_{i}+b y_{i}^{c}\right)}\right]^{v_{1}+v_{2}+v_{3}-1}\right\}
\end{aligned}
$$

The log-likelihood function can be obtained as:

$$
\begin{aligned}
& \mathcal{L}(\Phi)=m_{1} \ln \left(v_{2}\right)+\prod_{i=1}^{m_{1}} \ln \left(a+b c y_{2 i}^{c-1}\right)-\sum_{i=1}^{m_{1}}\left(a y_{2 i}+b y_{2 i}^{c}\right) \\
& +\left(v_{2}-1\right) \sum_{i=1}^{m_{1}} \ln \left[1-e^{-\left(a y_{2 i}+b y y_{2 i}^{c}\right)}\right]+m_{1} \ln \left(v_{1}+v_{3}\right)+ \\
& \sum_{i=1}^{m_{1}} \ln \left(a+b c y_{1 i}^{c-1}\right)-\sum_{i=1}^{m_{1}}\left(a y_{1 i}+b y_{1 i}^{c}\right)+\left(v_{1}+v_{3}-1\right) \times \\
& \sum_{i=1}^{m_{1}} \ln \left[1-e^{-\left(a y_{1 i}+b y_{1 i}^{c}\right)}\right]+m_{2} \ln \left(v_{1}\right)+\sum_{i=1}^{m_{2}} \ln \left(a+b c y_{1 i}^{c-1}\right)- \\
& \sum_{i=1}^{m_{2}}\left(a y_{1 i}+b y_{1 i}^{c}\right)+\left(v_{1}-1\right) \sum_{i=1}^{m_{2}} \ln \left[1-e^{-\left(a y_{1 i}+b y y_{i i}^{c}\right)}\right]+ \\
& m_{2} \ln \left(v_{2}+v_{3}\right)+\sum_{i=1}^{m_{2}} \ln \left(a+b c y_{2 i}^{c-1}\right)-\sum_{i=1}^{m_{2}}\left(a y_{2 i}+b y_{2 i}^{c}\right) \\
& +\left(v_{2}+v_{3}-1\right) \sum_{i=1}^{m_{2}} \ln \left[1-e^{-\left(a y_{2 i}+b y y_{2 i}^{c}\right)}\right]+m_{3} \ln \left(v_{3}\right)+ \\
& \sum_{i=1}^{m_{3}} \ln \left(a+b c y_{1}^{c-1}\right)-\sum_{i=1}^{m_{3}}\left(a y_{i}+b y_{i}^{c}\right)+\left(v_{1}+v_{2}+v_{3}-1\right) \\
& \sum_{i=1}^{m_{3}} \ln \left[1-e^{-\left(a y_{i}+b y_{i}^{c}\right)}\right] .
\end{aligned}
$$

The first partial derivatives of Eq. (43) with respect to $v_{1}, v_{2}, v_{3}$ and $c$ are given as in the following forms:

$$
\begin{aligned}
& \frac{\partial \mathcal{L}}{\partial v_{1}}=\frac{m_{1}}{v_{1}+v_{3}}+\sum_{i=1}^{m_{1}} \ln \left[1-e^{-\left(a y_{1 i}+b y_{1 i}^{c}\right)}\right]+\frac{m_{2}}{v_{1}}+ \\
& +\sum_{i=1}^{m_{2}} \ln \left[1-e^{-\left(a y_{1 i}+b y_{1 i}^{c}\right)}\right]+\sum_{i=1}^{m_{3}} \ln \left[1-e^{-\left(a y_{1 i}+b y_{1 i}^{c}\right)}\right]
\end{aligned}
$$




$$
\begin{aligned}
& \frac{\partial \mathcal{L}}{\partial v_{2}}=\frac{m_{1}}{v_{2}}+\sum_{i=1}^{m_{1}} \ln \left[1-e^{-\left(a y_{2 i}+b y_{2 i} c_{i}\right)}\right]+\frac{m_{2}}{v_{2}+v_{3}}+ \\
& \sum_{i=1}^{m_{2}} \ln \left[1-e^{-\left(a y_{2 i}+b y_{2 i}^{c_{i}}\right)}\right]+\sum_{i=1}^{m_{3}} \ln \left[1-e^{-\left(a y_{i}+b y_{i}^{c}\right)}\right] \text {, } \\
& \frac{\partial \mathcal{L}}{\partial v_{3}}=\frac{m_{1}}{v_{1}+v_{3}}+\sum_{i=1}^{m_{1}} \ln \left[1-e^{-\left(a y_{1 i}+b y_{1 i}^{c}\right)}\right]+\frac{m_{2}}{v_{2}+v_{3}}+ \\
& \sum_{i=1}^{m_{2}} \ln \left[1-e^{-\left(a y_{2 i}+b y_{2 i}^{c}\right)}\right]+\frac{m_{3}}{V_{3}}+\sum_{i=1}^{m_{3}} \ln \left[1-e^{-\left(a y_{i}+b y_{i}^{c}\right)}\right] \text {. } \\
& \frac{\partial \mathcal{L}}{\partial c}=b\left[\sum_{i=1}^{m_{1}} \mathcal{F}\left(y_{2 i}\right)+\left(v_{2}-1\right) \sum_{i=1}^{m_{1}} y_{2 i}^{c} \ln \left(y_{2 i}\right) \mathcal{A}\left(y_{2 i}\right)-\right. \\
& \sum_{i=1}^{m_{2}} y_{2 i}^{c} \ln \left(y_{2 i}\right)+\sum_{i=1}^{m_{1}} \mathcal{F}\left(y_{1 i}\right)+\left(v_{1}+v_{3}-1\right) \times \\
& \sum_{i=1}^{m_{1}} y_{1 i}^{c} \ln \left(y_{1 i}\right) \mathcal{A}\left(y_{1 i}\right)-\sum_{i=1}^{m_{1}} y_{1 i}^{c} \ln \left(y_{1 i}\right)+\sum_{i=1}^{m_{2}} \mathcal{F}\left(y_{1 i}\right)- \\
& \sum_{i=1}^{m_{2}} y_{1 i}^{c} \ln \left(y_{1 i}\right)+\left(v_{1}-1\right) \sum_{i=1}^{m_{2}} y_{1 i}^{c} \ln \left(y_{1 i}\right) \mathcal{A}\left(y_{1 i}\right)+ \\
& \sum_{i=1}^{m_{2}} \mathcal{F}\left(y_{2 i}\right)-\sum_{i=1}^{m_{2}} y_{2 i}^{c} \ln \left(y_{2 i}\right)+\left(v_{2}+v_{3}-1\right) \times \\
& \sum_{i=1}^{m_{2}} y_{2 i}^{c} \ln \left(y_{2 i}\right) \mathcal{A}\left(y_{2 i}\right)+\sum_{i=1}^{m_{3}} \mathcal{F}\left(y_{i}\right)-\sum_{i=1}^{m_{3}} y_{i}^{c} \ln \left(y_{i}\right) \\
& \left.+\left(v_{1}+v_{2}+v_{3}-1\right) \sum_{i=1}^{m_{3}} y_{i}^{c} \ln \left(y_{i}\right) \mathcal{A}\left(y_{i}\right)\right] \text {, }
\end{aligned}
$$

where:

$$
\mathcal{F}\left(y_{i}\right)=\frac{y_{i}^{c-1}\left[1+c \ln \left(y_{i}\right)\right]}{a+b c y_{i}^{c-1}}, \mathcal{A}\left(y_{i}\right)=\frac{e^{-\left(a y_{i}+b y_{i}^{c}\right)}}{1-e^{-\left(a y_{i}+b y_{i}^{c}\right)}} .
$$

Setting the first partial derivatives equal to zeros and solve the system of nonlinear equations with respect to $v_{1}, v_{2}, v_{3}$ and $c$ to get the MLEs. The Equations (44) to (47) are not easy to solve, so numerical technique is needed to get the MLEs.

Based on the asymptotic distributions of the MLEs, the approximate confidence intervals of the parameters $v_{1}, v_{2}, v_{3}$ and $c$ are derived. The variance-covariance matrix may be approximated by:

$S=I^{-1}$

where, $I$ is the information matrix as follows:

$$
I=E\left[\begin{array}{cccc}
-\frac{\partial^{2} \mathcal{L}}{\partial v_{1}^{2}} & -\frac{\partial^{2} \mathcal{L}}{\partial v_{1} \partial v_{2}} & -\frac{\partial^{2} \mathcal{L}}{\partial v_{1} \partial v_{3}} & -\frac{\partial^{2} \mathcal{L}}{\partial v_{1} \partial c} \\
-\frac{\partial^{2} \mathcal{L}}{\partial v_{2} \partial v_{1}} & -\frac{\partial^{2} \mathcal{L}}{\partial v_{2}^{2}} & -\frac{\partial^{2} \mathcal{L}}{\partial v_{2} \partial v_{3}} & -\frac{\partial^{2} \mathcal{L}}{\partial v_{2} \partial c} \\
-\frac{\partial^{2} \mathcal{L}}{\partial v_{3} \partial v_{1}} & -\frac{\partial^{2} \mathcal{L}}{\partial v_{3} \partial v_{2}} & -\frac{\partial^{2} \mathcal{L}}{\partial v_{3}^{2}} & -\frac{\partial^{2} \mathcal{L}}{\partial v_{3} \partial c} \\
-\frac{\partial^{2} \mathcal{L}}{\partial c \partial v_{1}} & -\frac{\partial^{2} \mathcal{L}}{\partial c \partial v_{2}} & -\frac{\partial^{2} \mathcal{L}}{\partial c \partial v_{3}} & -\frac{\partial^{2} \mathcal{L}}{\partial c^{2}}
\end{array}\right]
$$

The second partial derivatives can be obtained as follows:

$$
\begin{aligned}
& \frac{\partial^{2} \mathcal{L}}{\partial v_{1}^{2}}=-\frac{m_{1}}{\left(v_{1}+v_{3}\right)^{2}}-\frac{m_{2}}{v_{1}^{2}}, \frac{\partial^{2} \mathcal{L}}{\partial v_{1} \partial v_{2}}=0 \\
& \frac{\partial^{2} \mathcal{L}}{\partial v_{1} \partial v_{3}}=-\frac{m_{1}}{\left(v_{1}+v_{3}\right)^{2}}, \frac{\partial^{2} \mathcal{L}}{\partial v_{2}^{2}}=-\frac{m_{1}}{v_{2}^{2}}-\frac{m_{2}}{\left(v_{2}+v_{3}\right)^{2}}, \\
& \frac{\partial^{2} \mathcal{L}}{\partial v_{2} \partial v_{3}}=-\frac{m_{2}}{\left(v_{2}+v_{3}\right)^{2}}, \frac{\partial^{2} \mathcal{L}}{\partial v_{3}^{2}}=-\frac{m_{1}}{\left(v_{1}+v_{3}\right)^{2}}-\frac{m_{2}}{\left(v_{2}+v_{3}\right)^{2}}-\frac{m_{3}}{v_{3}^{2}}, \\
& \frac{\partial^{2} \mathcal{L}}{\partial c \partial v_{1}}=b\left[\sum_{i=1}^{m_{1}} y_{1 i}^{c} \ln \left(y_{1 i}\right) \mathcal{A}\left(y_{1 i}\right)+\sum_{i=1}^{m_{2}} y_{1 i}^{c} \ln \left(y_{1 i}\right) \mathcal{A}\left(y_{1 i}\right)+\sum_{i=1}^{m_{3}} y_{i}^{c} \ln \left(y_{i}\right) \mathcal{A}\left(y_{i}\right)\right] \text {, } \\
& \frac{\partial^{2} \mathcal{L}}{\partial c \partial v_{2}}=b\left[\sum_{i=1}^{m_{1}} y_{2 i}^{c} \ln \left(y_{2 i}\right) \mathcal{A}\left(y_{2 i}\right)+\sum_{i=1}^{m_{2}} y_{2 i}^{c} \ln \left(y_{2 i}\right) \mathcal{A}\left(y_{2 i}\right)+\sum_{i=1}^{m_{3}} y_{i}^{c} \ln \left(y_{i}\right) \mathcal{A}\left(y_{i}\right)\right] \text {, } \\
& \frac{\partial^{2} \mathcal{L}}{\partial c \partial v_{3}}=b\left[\sum_{i=1}^{m_{1}} y_{1 i}^{c} \ln \left(y_{1 i}\right) \mathcal{A}\left(y_{1 i}\right)+\sum_{i=1}^{m_{2}} y_{2 i}^{c} \ln \left(y_{2 i}\right) \mathcal{A}\left(y_{2 i}\right)+\sum_{i=1}^{m_{3}} y_{i}^{c} \ln \left(y_{i}\right) \mathcal{A}\left(y_{i}\right)\right] \text {, } \\
& \frac{\partial^{2} L}{\partial c^{2}}=\sum_{i=1}^{m_{1}}\left(\begin{array}{l}
\frac{2 b x_{2 i}^{c-1} \ln \left(y_{2 i}\right)+b c y_{2 i}^{c-1}\left(\ln \left(y_{2 i}\right)\right)^{2}}{a+b c y_{2 i}^{c-1}} \\
-\frac{\left(b y_{2 i}^{c-1}+b c y_{2 i}^{c-1} \ln \left(y_{2 i}\right)\right)^{2}}{\left(a+b c y_{2 i}^{c-1}\right)^{2}}
\end{array}\right)+\left(v_{2}-1\right) \times \\
& \sum_{i=1}^{m_{1}}\left(b y_{2 i}^{c}\left(\ln \left(y_{2 i}\right)\right)^{2} \mathcal{A}\left(y_{2 i}\right)-b^{2} y_{2 i}^{2 c}\left(\ln \left(y_{2 i}\right)\right)^{2}\left[A\left(y_{2 i}\right)+\mathcal{A}^{2}\left(y_{2 i}\right)\right]\right) \\
& +\sum_{i=1}^{m_{1}}\left(\begin{array}{l}
\frac{2 b y_{1 i}^{c-1} \ln \left(y_{1 i}\right)+b c y_{1 i}^{c-1}\left(\ln \left(y_{1 i}\right)\right)^{2}}{a+b c y_{1 i}^{c-1}} \\
-\frac{\left.b y_{1 i}^{c-1}+b c y_{1 i}^{c-1} \ln \left(y_{1 i}\right)\right)^{2}}{\left(a+b c y_{1 i}^{c-1}\right)^{2}}
\end{array}\right)+\left(v_{1}+v_{3}-1\right) \times
\end{aligned}
$$$$
\sum_{i=1}^{m_{1}}\left(b y_{1 i}^{c}\left(\ln \left(y_{1 i}\right)\right)^{2} A\left(y_{1 i}\right)-b^{2} y_{1 i}^{2 c}\left(\ln \left(y_{1 i}\right)\right)^{2}\right.
$$$$
\left.\left.\times\left[A\left(y_{1 i}\right)+A^{2}\left(y_{1 i}\right)\right]\right)-\sum_{i=1}^{m_{1}} b y_{2 i}^{c}\left(y_{2 i}\right)\right)^{2}-
$$$$
\sum_{i=1}^{m_{1}} b y_{2 i}^{c}\left(\ln \left(y_{1 i}\right)\right)^{2}-\sum_{i=1}^{m_{2}} b y_{1 i}^{c}\left(\ln \left(y_{1 i}\right)\right)^{2}+
$$$$
\sum_{i=1}^{m_{2}}\left(\begin{array}{l}
\frac{2 b y_{1 i}^{c-1} \ln \left(y_{1 i}\right)+b c y_{1 i}^{c-1}\left(\ln \left(y_{1 i}\right)\right)^{2}}{a+b c y_{1 i}^{c-1}} \\
-\frac{\left(b y_{1 i}^{c-1}+b c y_{1 i}^{c-1}\left(\ln \left(y_{1 i}\right)\right)^{2}\right.}{\left(a+b c y_{1 i}^{c-1}\right)^{2}}
\end{array}\right)+\left(v_{1}-1\right) \times
$$

$\sum_{i=1}^{m_{2}}\left(b y_{1 i}^{c}\left(\ln \left(y_{1 i}\right)\right)^{2} \mathcal{A}\left(y_{1 i}\right)-b^{2} y_{1 i}^{2 c}\left(\ln \left(y_{1 i}\right)\right)^{2} \times\left[\mathcal{A}\left(y_{1 i}\right)+\mathcal{A}^{2}\left(y_{1 i}\right)\right]\right)+$ $\sum_{i=1}^{m_{2}}\left(\begin{array}{l}\frac{2 b y_{2 i}^{c-1} \ln \left(y_{2 i}\right)+b c y_{2 i}^{c-1}\left(\ln \left(y_{2 i}\right)\right)^{2}}{a+b c y_{2 i}^{c-1}} \\ -\frac{\left(b y_{2 i}^{c-1}+b c y_{2 i}^{c-1}\left(\ln \left(y_{2 i}\right)\right)^{2}\right.}{\left(a+b c y_{2 i}^{c-1}\right)^{2}}\end{array}\right)+\left(v_{2}+v_{3}-1\right) \times$

$\sum_{i=1}^{m_{3}}\left(b y_{2 i}^{c}\left(\ln \left(y_{2 i}\right)\right)^{2} \mathcal{A}\left(y_{2 i}\right)-b^{2} y_{2 i}^{2 c}\left(\ln \left(y_{2 i}\right)\right)^{2} \times\left[\mathcal{A}\left(y_{2 i}\right)+\mathcal{A}^{2}\left(y_{2 i}\right)\right]\right)-$

$\sum_{i=1}^{m_{2}} b y_{2 i}^{c}\left(\ln \left(y_{2 i}\right)\right)^{2}-\sum_{i=1}^{m_{3}} b y_{i}^{c}\left(\ln \left(y_{i}\right)\right)^{2}+\left(v_{1}+v_{2}+v_{3}-1\right) \times$

$\sum_{i=1}^{m_{3}}\left(b y_{i}^{c}\left(\ln \left(y_{i}\right)\right)^{2} \mathcal{A}\left(y_{i}\right)-b^{2} y_{i}^{2 c}\left(\ln \left(y_{i}\right)\right)^{2} \times\left[\mathcal{A}\left(y_{i}\right)+\mathcal{A}^{2}\left(y_{i}\right)\right]\right)$

$+\sum_{i=1}^{m_{3}}\left(\frac{2 b y_{i}^{c-1} \ln \left(y_{i}\right)+b c y_{i}^{c-1}\left(\ln \left(y_{i}\right)\right)^{2}}{a+b c y_{i}^{c-1}}-\frac{\left(b y_{i}^{c-1}+b c y_{i}^{c-1}\left(\ln \left(y_{i}\right)\right)^{2}\right.}{\left(a+b c y_{i}^{c-1}\right)^{2}}\right)$.

By using Equation (48), we can compute the (1$\delta) 100 \%$ confidence interval for $v_{1}, v_{2}, v_{3}$ and $c$ respectively as: 
Table 1. American football league data

\begin{tabular}{lrrrrrrr}
\hline$Y_{1}$ & 2.05 & 9.05 & 0.85 & 3.43 & 7.78 & 10.57 & 7.05 \\
$Y_{2}$ & 3.98 & 9.05 & 0.85 & 3.43 & 7.78 & 14.28 & 7.05 \\
$Y_{1}$ & 2.58 & 7.23 & 6.85 & 32.45 & 5.78 & 13.80 & 7.25 \\
$Y_{2}$ & 2.58 & 9.68 & 34.58 & 42.35 & 25.98 & 49.75 & 7.25 \\
$Y_{1}$ & 4.25 & 1.65 & 6.42 & 4.22 & 15.53 & 2.90 & 7.02 \\
$Y_{2}$ & 4.25 & 1.65 & 15.08 & 9.48 & 15.53 & 2.90 & 7.02 \\
$Y_{1}$ & 6.42 & 10.4 & 0.75 & 3.88 & 0.75 & 11.63 & 1.38 \\
$Y_{2}$ & 6.42 & 10.25 & 2.98 & 6.43 & 0.75 & 17.37 & 1.38 \\
$Y_{1}$ & 10.53 & 12.13 & 14.58 & 11.82 & 5.52 & 31.13 & 14.58 \\
$Y_{2}$ & 10.53 & 12.13 & 14.58 & 11.82 & 11.27 & 49.88 & 20.57 \\
$Y_{1}$ & 10.15 & 8.87 & 17.83 & 10.85 & 8.53 & 8.98 & 19.65 \\
$Y_{2}$ & 10.15 & 8.87 & 17.83 & 38.07 & 14.57 & 8.98 & 10.70 \\
\hline
\end{tabular}

Table 2. The MLEs of the parameters

\begin{tabular}{lllllll}
\hline Models & $\hat{v}_{2}$ & $\hat{v}_{2}$ & $\hat{v}_{3}$ & $\hat{a}$ & $\hat{b}$ & \multicolumn{1}{c}{} \\
\hline BGED & 0.0500 & 0.1700 & 0.3640 & 0.012 & -- & - \\
BGLFR & 0.0294 & 0.1701 & 0.3705 & 0.012 & $2.159 \times 10^{-8}$ & - \\
BEMW & 0.0298 & 0.1777 & 0.3745 & 0.012 & $2.159 \times 10^{-8}$ & 4.7314 \\
\hline
\end{tabular}

Table 3. The values of $\mathcal{L}, \mathrm{AIC}, \mathrm{AICC}$ and BIC

\begin{tabular}{lllll}
\hline Models & $\mathcal{L}$ & AIC & AICC & BICB \\
\hline GED & -354.885 & 711.770 & 711.870 & 713.507 \\
BGLFR & -270.719 & 547.439 & 548.070 & 552.652 \\
BEMW & -262.407 & 532.814 & 533.895 & 539.765 \\
\hline
\end{tabular}

$v_{i} \pm z_{\frac{\delta}{2}} \sqrt{S_{i i}}, i=1,2,3, c \pm z_{\frac{\delta}{2}} \sqrt{S_{44}}$

where, $z_{\delta / 2}$ is the upper $(\delta / 2)$ th percentile of the standard normal distribution.

\section{Data Analysis}

The following data represent the American Football. For more details Csorgo and Welsh (1989). The data (scoring times in minutes and seconds) are represented in the following Table 1.

The MLEs of the unknown parameters of $B G E D\left(\hat{v}_{1}, \hat{v}_{2}, \hat{v}_{3}, \hat{a}\right) \quad$ Kundu and Gupta (2009), $\operatorname{BGLFRD}\left(\hat{v}_{1}, \hat{v}_{2}, \hat{v}_{3}, \hat{a}, \hat{b}\right)$, Sarhan et al. (2011) and $\operatorname{BEMW}\left(\hat{v}_{1}, \hat{v}_{2}, \hat{v}_{3}, \hat{a}, \hat{b}, \hat{c}\right)$ models are presented in Table 2.

Table 3 contains the values of $\mathcal{L}$, AIC, AICC and BIC for the three models.

Based on the Table 3, the BEMW model fits the data better than the BGED and BGLFR model. Also, by substituting the MLEs of unknown parameters in Equation (48), the variance covariance matrix can be estimated as follows:

$\left(\begin{array}{cccc}0.00043193 & 0.00000213 & -0.00015334 & 0.00001292 \\ 0.00000213 & 0.00195113 & -0.00004533 & 0.00017073 \\ -0.00015334 & -0.00004533 & 0.00368595 & 0.00013815 \\ 0.00001292 & 0.00017073 & 0.00013815 & 0.01346693\end{array}\right)$

The approximate $95 \%$ confidence interval of $v_{1}, v_{2}$, $v_{3}$ and $c$ are [0, 0.070535], [0.091124, 0.264276], $[0.255504,0.493496]$ and $[4.503948,4.958852]$, respectively.

\section{Conclusion}

In this study, the BEMWD whose marginals are EMW distributions is introduced. We derive some statistical and reliability measures of the new bivariate distribution. The reliability estimation for the stressstrength model is obtained. Maximum likelihood estimates are discussed. Moreover, the observed variance covariance matrix is derived. The real data set is analyzed. Finally, we conclude that, the new bivariate model fits the given real data best than the BGED and BGLFR models.

\section{Acknowledgement}

The authors are grateful to the Editor and referees for a careful checking of the details and for helpful comments that improved this paper.

\section{Author's Contributions}

A. Mustafa and M. Mahmoud: with the consultation of each other carried out this work and drafted the manuscript together. Both authors read and approved the final manuscript. 


\section{Ethics}

The author declares that there is no conflict of interests regarding the publication of this article.

\section{References}

Al-Khedhairi, A. and A. El-Gohary, 2011. A new class of bivariate Gompertz distributions. Int. J. Math. Anal., 2: 235-253.

Basu, A.P., 1971. Bivariate failure rate. Am. Statist. Assoc., 66: 103-104.

Csorgo, S. and A.H. Welsh, 1989. Testing for exponential and Marshall-Olkin distribution. J. Statistical Planning Inference, 23: 287-300.

Elbatal, I., 2011. Exponentiated modified weibull distribution. Econom. Quality Control, 26: 189-200.

El-Sherpieny, E.A., S.A. Ibrahim and R.E. Bedar, 2013. A new bivariate generalized Gompertz distribution. Asian J. Applied Sci., 1: 2321-0893.

Johnson, N.L. and S. Kotz, 1975. A vector multivariate hazard rate. J. Multivariate Anal., 5: 53-66. DOI: 10.1016/0047-259X(75)90055-X
Kundu, D. and R.D. Gupta, 2009. Bivariate generalized exponential distribution. J. Multivariate Anal., 100: 581-593. DOI: 10.1016/j.jmva.2008.06.012

Kundu, D. and K. Gupta, 2013. Bayes estimation for the Marshall-Olkin bivariate Weibull distribution. J. Computat. Statistics Data Anal., 57: 271-281.

Marshall, A.W. and I.A. Olkin, 1986. A multivariate exponential distribution. J. Am. Statistical Assoc., 62: 30-44. DOI: 10.2307/2282907

Mustafa, A., 2016. A new bivariate distribution with generalized quadratic hazard rate Marginals. J. Math. Statistics, 12: 255-270.

Sarhan, A. and N. Balakrishnan, 2007. A new class of bivariate distributions and its mixture. J. Multivariate Anal., 98: 1508-1527.

Sarhan, A. and M. Zaindin, 2009. Modified Weibull distribution. Applied Sci., 11: 123-136.

Sarhan, A., D. Hamilton, B. Smith and D. Kundu, 2011. The bivariate generalized linear failure rate distribution and its multivariate extension. Computat. Statistics Data Anal., 55: 644-654. DOI: $10.1016 /$ j.csda.2010.06.006 